\title{
CARACTÉRISTIQUES ENTREPRENEURIALES ET DÉCISION DU DIRIGEANT-CRÉATEUR D'ENTREPRISE
}

\begin{abstract}
Concernant les décisions au sein des organisations, il y a celle particulière de créer sa propre entreprise et d'en assurer le fonctionnement et la pérennité. Il est alors indispensable de s'intéresser aux caractéristiques entrepreneuriales du créateur en tant que déterminants de ses décisions. Les travaux relatifs à l'inventaire des caractéristiques entrepreneuriales (Gasse, 1996; 2003) nous offre cette possibilité. Celles-ci permettent de cerner le potentiel entrepreneurial du créateur. Mais une recherche plus approfondie conduit à les exploiter comme déterminants de la décision d'être dirigeant créateur. C'est cette option qui fait l'objet de notre article. Après avoir identifié les caractéristiques des entrepreneurs sur la manière d'opérer le choix de créer une entreprise à partir de leurs propres perceptions ou d'image de soi. Cette démarche a été adaptée à une enquête réalisée auprès de 172 dirigeants créateurs de PME en activité avec une perspective de pérennité au dernier trimestre 2009. La finalité essentielle d'une telle enquête est d'inventorier et de classer les caractéristiques entrepreneuriales et, par la suite, d'établir une typologie des entrepreneurs. Compte tenu des caractéristiques de l'enquête, l'analyse consiste à différencier la perception de leur décision de créateur d'entreprise.

Les résultats de notre étude nous conduisent à trois catégories d'entrepreneurs au vu de leurs opinions sur les caractéristiques déterminant leurs comportements décisionnels. Nous rapprochons ces résultats aux travaux de Baumol (1968). Ainsi nous pouvons positionner les décideurs non routiers entre les créateurs gestionnaires et les créateurs entrepreneurs. Le cheminement que peut effectuer le créateur gestionnaire (ou exclusivement routinier) vers le créateur entrepreneur (ou véritablement non routinier) incite à la mise en place de pédagogies d'accompagnement plus adaptées.
\end{abstract}

Les mots clés: Entrepreneuriales, Motivations,Décision, Valeurs, Entrepreneuriat

\section{INTRODUCTION}

Concernant les décisions au sein des organisations, il y a celle particulière de créer sa propre entreprise et d'en assurer le fonctionnement et la pérennité. Dans ses travaux Schumpeter $(1936 ; 1942)$ a qualifié l'entrepreneur comme «le pivot autour duquel tout tourne». Aujourd'hui, les chercheurs affirment que nous sommes dans «l'ère de l'entrepreneur» (Goffee et Scase, 1987); on s'intéresse aux caractéristiques entrepreneuriales du créateur et aux déterminants de l'entrepreneuriat. En fait, les nouvelles entreprises sont perçues comme une source de nouveaux emplois et, finalement, comme le pivot de la croissance et du développement économique (Blanchflower et Oswald, 1990). Ceci justifie l'abondance de travaux publiés sur l'esprit d'entreprise au

\footnotetext{
${ }^{1}$ Gérard A. Kokou Dokou, Maître de Conférences à l'Université du Littoral Côte d'Opale, (Département Techniques de Commercialisation de l'IUT du Littoral), France. Directeur du Centre Entrepreneuriat du Littoral (CEL) et de son laboratoire CEL-LAB. 252, Avenue de l'Université, 59383 Dunkerque cedex 01, France, e-mail : gdokou@ nordnet.fr
} 
cours des vingt dernières années (Acs et Audretsch, 2003; Audretsch, 2002; Casson, 1982, 2003; Gartner, 1990;Glancey et McQuaid, 2000; Shane, 2000; Storey, 2000; Swedberg, 2000; Westhead et Wrigth, 2000; etc). Bull et al. (1996) suggèrent cinq grandes catégories de publications: la littérature qui ne s'intéresse pas directement à la théorie, mais qui se concentre sur la tentative de donner une définition au mot «Entrepreneur», l'analyse des traits psychologiques des personnes définies comme des entrepreneurs, des enquêtes sur la formation de nouvelles entreprises, des études sur les stratégies reconnues comme essentielles afin d'expliquer le succès des entreprises commerciales, et les œuvres sur les facteurs environnementaux qui influent sur les actions de l'entreprise. Cependant, l'esprit d'entreprise est toujours «l'une des formes de comportement économique les plus insaisissables et moins comprises» (Eboli, 1997). D'où la nécessité d'identifier les caractéristiques entrepreneuriales des créateurs d'entreprise. C'est l'optique des travaux relatifs à l'inventaire des caractéristiques entrepreneuriales (Gasse, 1996; 2003). Celles-ci permettent de cerner le potentiel entrepreneurial du créateur mais aussi les déterminants de sa décision d'être dirigeant créateur. C'est cette dernière option qui fait l'objet de notre article.

Comme le suggère Baumol (1968), il est nécessaire de cerner la position de l'entrepreneur pour comprendre le comportement de son entreprise. D'où la distinction entre le créateur gestionnaire et le créateur entrepreneur. Le premier supervise les activités en cours: la répartition des investissements, les contrôles des horaires et les contrats à respecter, etc. Il est en charge de l'activité de routine. Inversement, la fonction d'entrepreneur concerne l'élaboration et la mise en œuvre de nouvelles idées: il est «l'innovateur de Schumpeter» qui perçoit les besoins non satisfaits (Kirzner, 1983). Il travaille plus dur que le gestionnaire (De Fraja, 1996). Il fournit de nouvelles idées, de nouveaux produits et de nouvelles manières de faire les choses (Iyigun et Owen, 1998). La distinction entre l'entrepreneur et le gestionnaire reflète les deux types de fonctions sous-tendant le choix d'être dirigeant créateur: les décisions de routine et les décisions non courantes. Peut-on trouver une telle distinction à partir des caractéristiques entrepreneuriales particulières selon les types de créateurs?

Pour répondre à cette interrogation, il nous paraît intéressant d'observer les caractéristiques des entrepreneurs sur la manière d'opérer le choix de créer une entreprise à partir de ses propres perceptions ou d'image de soi. L'approche retenue est celle de l'adaptation de l'Inventaire des Caractéristiques Entrepreneuriales (ICE) du professeur Yvon GASSE (1996; 2003). Cette démarche a été adaptée à une enquête réalisée auprès de 172 dirigeants créateurs de PME en activité avec une perspective de pérennité au dernier trimestre 2009.

Nos questionnements fondamentaux sont simples, voire triviaux. On peut, en effet, se demander s'il existe ou non un ou plusieurs profil(s) type(s) de dirigeants créateurs de PME à partir de leurs caractéristiques entrepreneuriales. Il s'agit également de savoir si l'entrepreneur appartient à une certaine catégorie sociale ou familiale ou à un profil psychologique précis l'aidant dans sa décision de devenir dirigeant créateur. Enfin, on est en droit de chercher si l'élaboration d'un tel profil peut aider à combler certaines lacunes d'un porteur de projet de création d'entreprise. Nous avons diligenté une enquête sur les caractéristiques entrepreneuriales auprès de 172 créateurs de PME du littoral dunkerquois 2009 (Nord de la France) au dernier trimestre 2009. La finalité essentielle d'une telle enquête est d'inventorier et de classer les caractéristiques entrepreneuriales et, par la suite, d'établir une typologie des entrepreneurs. Compte tenu des caractéristiques de l'enquête, 
l'analyse consiste à différencier la perception de leur décision de créateur d'entreprise Autrement dit, deux objectifs principaux sous-tendent cette étude.

- Cerner les caractéristiques entrepreneuriales qui accompagnent la décision de devenir dirigeant créateur d'entreprise; les composantes d'une telle décision relèvent des représentations, a posteriori, que le dirigeant créateur se fait de ses propres caractéristiques entrepreneuriales.

- Déterminer s'il y a des différenciations entre les entrepreneurs au regard des modes décisionnels mis en évidence.

C'est une phase d'étude qui aidera à comprendre les personnalités des entrepreneurs et permettra d'apporter les premiers éléments de réponse à ces deux objectifs relatifs à une meilleure connaissance des entrepreneurs face aux modes décisionnels. La démarche méthodologique retenue (voir encadré) nous permet d'articuler l'article autour de trois points.

- Importance des caractéristiques entrepreneuriales dans la décision du dirigeant créateur.

- Pertinence des interactions entre le comportement entrepreneurial du dirigeant créateur et ses caractéristiques intrinsèques.

- Positionnement des caractéristiques entrepreneuriales dans les décisions de routine et les choix non courants au regard des représentations que le dirigeant se fait de son contexte d'action.

\section{APPROCHES METHODOLOGIQUES}

Les approches méthodologiques de cette étude sont fondées sur les méthodes statistiques de traitement d'une base de données d'enquête. Partant des résultats de la base d'enquête mise en place, il s'agit d'extraire les informations essentielles en utilisant les techniques statistiques d'analyse exploratoire multidimensionnelle et d'établir une première vue d'ensemble sur les liens éventuels entre les identifications des entrepreneurs et leurs perceptions du choix de créateur d'entreprise.

\section{Description de la base de données d'enquête}

Afin de mener à bien cette étude, un questionnaire a été rempli par 172 créateurs de PME du littoral dunkerquois. Il s'agit ici d'identifier et d'établir la typologie des entrepreneurs. $\mathrm{Ce}$ questionnaire se décompose en deux parties. Une première partie concerne les identifiants des entrepreneurs enquêtés: nom, prénom, coordonnées, sexe, âge, plus haut degré de scolarité, catégorie socioprofessionnelle et l'expérience professionnelle avant la création d'entreprise. La deuxième partie du questionnaire est composée de 120 questions (variables) relatives aux quinze types de caractéristiques entrepreneuriales. Il s'agit de quinze dimensions clés articulées autour de cinq axes.

Axe des motivations : accomplissement, pouvoir et autonomie ;

Axe des aptitudes : confiance en soi, énergie, persévérance, tolérance au stress et capacité conceptuelle (imagination, intuition conceptuelle);

Axe des intérêts: innovation (aimer et réaliser des innovations) et action (faire agir et faire soi-même) ;

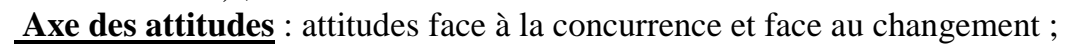


Axe du comportement : affectivité (s'intéresser aux autres, écouter et apprécier les autres), cognition (veille informationnelle et recherche de nouvelles connaissances) et coaction (conception et réalisation de projet, saisie d'opportunités).

Chacune des 120 questions est codée en 4 modalités mutuellement exclusives qui vont de la modalité de totalement en accord à la modalité de totalement en désaccord selon une échelle de Likert. C'est dire que quinze nouvelles variables (dites variables clés: composantes des cinq axes ci-dessus) ont été générées à partir des 120 questions. Chaque variable clé est obtenue en calculant un score moyen des réponses des dirigeants ayant répondu au questionnaire écrit administré en face à face.

\section{Méthodes de traitement}

Après recodage de certaines variables de la base d'enquête et suppression des informations manquantes, nous disposons d'une base de données qualifiée comportant 172 dirigeants créateurs de PME évalués selon 8 variables d'identification et 95 variables de perception. Cette base de données sous Excel a par la suite été importée sous SPAD et sous SPSS. Au-delà de cette base de données sous Excel, nous disposons ainsi de deux versions de la même base de données d'enquête permettant d'avoir des traitements statistiques plus complets pour l'analyse.

Deux types de traitement statistiques ont été menés. Un premier traitement a consisté en une analyse exploratoire simple afin de décrire les différentes variables de l'enquête. Un deuxième traitement a conduit à une analyse statistique multiple. Cette dernière analyse est obtenue grâce aux méthodes statistiques exploratoires multidimensionnelles (Analyse des Correspondances Multiples (ACM) et Classification Ascendante Hiérarchique (CAH)) disponibles dans le logiciel SPAD. Il s'agit essentiellement d'extraire les grandes tendances de perception de soi des dirigeants créateurs de PME et de les regrouper dans un certain nombre de classes d'entrepreneurs.

\section{Indications sur les caractéristiques des dirigeants enquêtés}

Tableau1. Quelques indications sur l'histoire de vie des dirigeants de PME enquêtés

\begin{tabular}{|c|c|c|c|c|c|c|c|c|}
\hline \multicolumn{2}{|c|}{ Sexe } & \multicolumn{7}{|c|}{ Age } \\
\hline Hommes & Femmes & \multirow{2}{*}{$\begin{array}{l}\text { Moins } \\
\text { de 25 } \\
\text { ans } \\
\end{array}$} & \multirow{2}{*}{$\begin{array}{l}25 / 29 \\
\text { ans }\end{array}$} & \multirow{2}{*}{$\begin{array}{l}\text { 30/34 } \\
\text { ans } \\
\end{array}$} & \multirow[t]{2}{*}{$35 / 39$ ans } & \multirow[t]{2}{*}{$40 / 44$ ans } & \multirow[t]{2}{*}{$45 / 49$ ans } & \multirow{2}{*}{$\begin{array}{l}50 \text { ans et } \\
\text { plus }\end{array}$} \\
\hline & & & & & & & & \\
\hline 69\% & $31 \%$ & $3 \%$ & $8 \%$ & $10 \%$ & $16 \%$ & $20 \%$ & $18 \%$ & $25 \%$ \\
\hline \multicolumn{9}{|c|}{ Niveau scolaire et expérience professionnelle } \\
\hline $\begin{array}{l}\text { Etudes } \\
\text { primaires }\end{array}$ & $\begin{array}{l}\text { BEPC ou } \\
\text { niveau }\end{array}$ & $\begin{array}{l}\text { Bac ou } \\
\text { niveau }\end{array}$ & Bac+2 & $\begin{array}{l}\text { Bac+3 } \\
\text { et plus }\end{array}$ & $\begin{array}{l}\text { Parents } \\
\text { entrepreneurs }\end{array}$ & $\begin{array}{l}\text { Parents non } \\
\text { entrepreneurs }\end{array}$ & \begin{tabular}{|l|} 
Expérience \\
de salarié
\end{tabular} & $\begin{array}{l}\text { Aucune } \\
\text { expérience }\end{array}$ \\
\hline & & & & & indépendants & indépendants & PME & en PME \\
\hline $6 \%$ & $45 \%$ & $17 \%$ & $21 \%$ & $11 \%$ & $47 \%$ & $53 \%$ & $63 \%$ & $37 \%$ \\
\hline
\end{tabular}

Les hommes représentent près des deux tiers des dirigeants de PME enquêtés (69\%) tandis que les femmes en représentent le tiers (31\%). Les dirigeants de plus de 50 ans ont le plus fort pourcentage par rapport aux autres tranches d'âge. Ainsi, $79 \%$ de nos 
entrepreneurs ont plus de 35 ans. Nous observons que plus de la moitié de ces individus ont plus de 40 ans et qu'aucun n'a moins de 20 ans.

Près de la moitié de ce groupe de responsables a le BEPC et au-delà. Compte tenu de la répartition de l'âge de ces entrepreneurs, nous pourrions penser que les individus les plus âgés soient les moins diplômés et inversement: que les plus jeunes soient les plus diplômés.

On remarque également que $47 \%$ des parents des personnes enquêtées possèdent ou ont possédé une entreprise ou ont déjà travaillé à leur propre compte c'est-à-dire près d'une personne sur deux. L'expérience de salarié dans une PME est une réalité pour $63 \%$ d'entre eux. Plus d'un entrepreneur sur deux (59\%) a créé une micro-entreprise (de 1 à 9 salariés), un tiers $(34 \%)$ est plutôt chef d'une petite entreprise (de 10 à 49 salariés) et $7 \%$ ont créé une entreprise moyenne (de 50 à 249 salariés) $)^{2}$.

\section{POIDS DES CARACTERISTIQUES ENTREPRENEURIALES DES REPRESENTATIONS SYMBOLIQUES DANS LA CREATION D'ENTREPRISE}

Ces caractéristiques constituent les items proposés aux dirigeants enquêtés. Ils les ont évaluées à partir de leurs propres représentations. Rappelons que la notion de représentation sociale, introduite au début des années soixante par Serge MOSCOVICI (1961), confère à l'action et au contexte social un rôle heuristique significatif dans l'appréciation des choix et des comportements effectifs des acteurs engagés dans la vie sociale et organisationnelle, notamment dans la création d'activité ou d'entreprise. Elle renvoie aux interactions sujet/objet représenté dans un contexte socio-économique mouvant.

Il n'est pas inutile de préciser que dès 1898 Emile DURKHEIM (1898) donne à la notion de représentation ses dimensions sociologiques et psychologiques contemporaines. En effet, les développements les plus récents de la psychologie sociale sont venus confirmer l'importance des éléments subjectifs qui naissent dans le quotidien des interactions sociales et qui sont partiellement indépendants des structures et des processus cognitifs individuels. Partiellement indépendants, parce que la compréhension, la construction et la production de sens, qui guident l'action, ne résultent pas uniquement d'activités purement cognitives. Des nombreuses et complexes interactions qui caractérisent un champ socioéconomique tel que la création d'entreprise émergent des discours, des pratiques, des idées, des valeurs, des idéologies, des cultures et des schèmes de pensée qui sous-tendent les conduites des entrepreneurs et donnent sens à leurs comportements. Les décisions qu'ils prennent ou les choix qu'ils sont amenés à effectuer relèvent d'une rationalité située et construite (Zaoual, 1996). Celle-ci intègre objectivité et subjectivité, rationalité et irrationalité ou certitude et incertitude à travers leurs caractéristiques entrepreneuriales.

Ainsi, le champ de représentations de l'entrepreneur se construit et se restructure en permanence sous l'influence d'une multitude de facteurs psychosociologiques, cognitifs et symboliques qui se combinent, se croisent et accentuent la complexité des systèmes de représentations par le jeu des interactions. Tandis que, la notion d'interaction dynamique et récursive, qu'introduit la pensée complexe appliquée à la création et à la gestion des entreprises, nous fait découvrir un sujet connaissant (le dirigeant créateur) agissant et agi

\footnotetext{
${ }^{2}$ Nos entreprises moyennes ont plutôt entre 50 et 120 salariés
} 
par les relations physiques et virtuelles qu'il entretient avec le monde qui l'entoure et qu'il filtre grâce aux caractéristiques entrepreneuriales. Celles-ci sont articulées autour des cinq dimensions qu'il convient de rappeler. Il s'agit des motivations, des aptitudes, des intérêts, des attitudes et du comportement entrepreneurial ${ }^{3}$.

\subsection{Du rôle des motivations}

A propos de «l'accomplissement», les entrepreneurs sont en grande majorité motivés de mener à terme les projets exigeants, d'être meilleurs que les autres dans leur domaine, d'avoir le plein contrôle des ressources nécessaires au travail mené, de vouloir que tout travail comporte de nouveaux savoirs et que les projets retenus aient de la valeur. Ils ont presque tous une volonté absolue à fixer leurs propres objectifs. Mais un tiers d'entre eux, opposé au reste, tolère aussi bien que quiconque l'inefficacité, n'a pas une volonté absolue d'accomplir les choses difficiles et préfère choisir des amis plutôt que des experts comme partenaires de la conduite d'un projet.

Concernant «le pouvoir», la majorité des entrepreneurs enquêtés sont enclins à être patron, pensent être meilleur que les autres dans leur domaine, ont une préférence pour les travaux à fortes responsabilités, influencent les autres et les amènent, autant que possible, à faire ce qu'ils souhaitent. Il apparaît également qu'un tiers de ces entrepreneurs ne se conforme pas facilement aux directives reçues et est très peu motivé pour diriger des groupes de travail. Pour «l'autonomie» deux tiers des entrepreneurs ont une préférence pour décider seul, ne se conforment pas facilement aux directives reçues, ont un sentiment de gêne par des contraintes et des restrictions obligatoires et préfèrent les activités n'obligeant pas des directives préétablies. Ils sont dans l'ensemble à l'aise pour prendre des décisions autonomes et en grande majorité, considèrent qu'avoir une bonne marge de manœuvre est une source d'efficacité. Mais ces mêmes entrepreneurs sont attachés au respect des conventions; ils sont également prêts à accepter les démarches des autres au détriment de leurs propres méthodes. De ce fait, l'autonomie est une caractéristique entrepreneuriale évaluée favorablement par les dirigeants enquêtés avec l'apparition de certaines contradictions.

Dans l'ensemble, les dirigeants créateurs sont concordants au niveau des composantes de l'accomplissement et du pouvoir et sont plutôt divergents sur celles de l'autonomie. Il apparaît donc que ces responsables soient essentiellement motivés par le besoin d'accomplissement et par le pouvoir exercé sur les autres en tant que chef d'entreprise. C'est déterminant dans leur choix d'être dirigeant créateur d'entreprise.

\subsection{La persévérance : la clef de la délivrance entrepreneuriale}

De par la variable «confiance en soi», les dirigeants créateurs enquêtés ont une aisance dans l'expression de leurs opinions, ont confiance dans la réussite de toute activité entreprise et se sentent à la hauteur de leur tâche. Ils sont à l'aise dans les discussions avec leurs confrères même en cas de désaccord et sont certains de mener à bien un projet planifié. Mais un tiers des entrepreneurs n'est pas à l'aise devant les personnes en autorité, ne juge pas obtenir de bons résultats par rapport aux moyens décidés et n'est pas à l'aise dans la prise de parole devant un groupe ni dans la prise de décisions.

Il en est de même de la variable «énergie». Nos dirigeants créateurs de PME ont, dans

\footnotetext{
${ }^{3}$ Les opinions positives développées par la suite sont émises par plus des deux tiers des enquêtés
} 
l'ensemble, une volonté de travail de longs moments sans interruption et sont toujours en situation de forte activité. Seul un tiers d'entre eux ne peut accomplir des tâches multiples et ne pense pas disposer de beaucoup plus d'énergie que les autres. Concernant la «persévérance», ils ont une volonté d'aller jusqu'au bout de la tâche à accomplir peu importent les obstacles ou les difficultés. Ils fournissent un maximum d'efforts et travaillent de longues heures sans interruption pour la réussite d'un projet entrepris et refusent l'échec. Mais ils doivent gérer leur «stress» pour maintenir leur niveau de productivité et leur sérénité devant des conditions de travail difficiles ou face à la complexité de multiples tâchent à accomplir. Par ailleurs, ils s'adaptent facilement face à des changements importants et ont une vision toujours positive des choses. La «capacité conceptuelle»y joue un rôle important. Ainsi, les entrepreneurs enquêtés ont en grande majorité une vision nette des résultats avant le démarrage de toute opération, une volonté de participation à des travaux nécessitant un ajustement à des compétences différentes. Ils considèrent qu'il est plus efficace de résoudre un problème avec la prise en compte de plusieurs choix. Ils ne prennent uniquement en compte que les grandes étapes d'un projet. Ces mêmes deux tiers des enquêtés empruntent fréquemment des exemples à d'autres champs d'activité et sont habiles à dénouer des situations très complexes.

Les réponses des entrepreneurs enquêtés sont essentiellement en accord avec les composantes de la persévérance tandis qu'elles sont plus divisées sur celles de la confiance en soi, de l'énergie, de la tolérance au stress et de la capacité conceptuelle. Ainsi, la persévérance est la seule aptitude entrepreneuriale sur laquelle plus des deux tiers des dirigeants créateurs de PME ont un avis convergent.

\subsection{Les intérêts : innovation et création}

Les enquêtés, dans l'ensemble, imaginent constamment d'autres méthodes pour mieux exercer leur métier de dirigeant, sont enclins à produire des idées originales et pensent important de suivre les projets de leur début jusqu'à leur fin. Un tiers de ces entrepreneurs ne génère pas constamment des idées nouvelles, n'imaginent pas de multiples utilisations à des objets d'usage courant et ne sont guère attentifs aux nouveautés hors de leur champ d'activité. Etre le premier à essayer les nouveaux produits qui sortent sur le marché n'est pas un critère déterminant, selon eux, pour être dirigeant créateur car seulement la moitié de ces entrepreneurs pensent innovateurs.

Environ un créateur sur deux accepterait de recommencer l'aventure entrepreneuriale en cas d'échec. Dans leurs réponses, deux tiers des entrepreneurs agissent sous l'impulsion du moment quitte à se réajuster par la suite. En majorité, ils sont impatients lors de réunions tardant à entrer dans le vif du sujet, à l'aise et prennent du plaisir à être au cœur de l'action.

On se rend compte que les réponses des entrepreneurs présentent des avis divergents sur certaines composantes de l'intérêt pour l'innovation et l'action.

\subsection{Les attitudes: compétition et création}

«Face à la concurrence», la compétition est majoritairement considérée comme un stimulant d'efficacité dans les affaires. C'est un des ressorts essentiels du métier de dirigeant créateur de PME. Dans l'ensemble, les entrepreneurs ont une facilité «d'adaptation aux changements» importants. Mais ils trouvent les contraintes et les restrictions auxquelles il faut se plier comme contraires à leur volonté d'agir. Au même 
moment, ils avouent avoir une volonté de participation à des travaux nécessitant un ajustement à des compétences différentes avec la possibilité d'introduire des changements à tous les niveaux de leur entreprise.

De telles réponses montrent que les entrepreneurs semblent avoir une perception commune sur leurs attitudes face à la concurrence et au changement.

\subsection{Le comportement entrepreneurial : perception positivement convergente}

Il s'agit des opinions que les enquêtés ont émis sur leurs propres comportements. Ils déclarent se sentir à l'aise dans les discussions discordantes avec leurs confrères et avouent disposer d'un talent de mobilisateur. Ils sont profondément ancrés dans leurs rôles et ont du mal à se mettre à la place des autres. Le poids de la variable «affectivité» est donc limité. Ce n'est pas le cas de la «cognition». En effet, une grande majorité des entrepreneurs pensent importantes les méthodologies d'action débouchant rapidement sur des résultats, consultent régulièrement les publications liées à leur champ d'activité, pensent possible de retirer quelque chose d'intéressant de n'importe quelle situation, ont une volonté de participation à des travaux nécessitant un ajustement à des compétences différentes. Par ailleurs, ils estiment que les projets retenus doivent avoir de la valeur, que les expériences concrètes sont une meilleure source d'apprentissage, et qu'il est plus efficace de résoudre un problème avec la prise en compte de plusieurs choix. De plus, ils considèrent les résultats issus des phases de mise en œuvre de projet comme une source de nouvelles connaissances. Enfin, ils sont presque unanimes à considérer que les résultats obtenus constituent une source d'évaluation des progrès personnels et de l'efficacité du système de veille informationnelle. C'est aussi le point d'ancrage de leur «comportement dans l'action». Ainsi, les enquêtés avouent faire preuve d'une volonté constante de saisir les occasions susceptibles de se présenter et de prise d'initiative. Pour eux, toutes les circonstances peuvent être source de profit. C'est pour cela qu'il leur est indispensable d'être à l'aise au niveau du travail nécessitant des décisions rapides et des contacts avec des personnes ressources dans la mise en œuvre des projets de l'entreprise. De la même manière, deux tiers d'entre eux pensent que l'intuition a un fort impact sur les décisions prises. Ils sont ainsi amenés à agir sous l'impulsion du moment avec réajustement par la suite même s'ils pensent avoir de l'influence ou une bonne marge de manœuvre sur leurs événements personnels.

\section{INTERACTIONS ENTRE LE COMPORTEMENT ENTREPRENEURIAL DU DIRIGEANT CREATEUR ET SES CARACTERISTIQUES INTRINSEQUES}

Après la phase d'exploration simple, la base de données qualifiée a été soumise à une chaîne d'analyses exploratoires multidimensionnelle utilisant les 8 éléments d'identification et les 95 questions non agrégées de l'enquête. Le but principal de cette chaîne d'approches exploratoires est de rechercher et de structurer les grandes tendances des perceptions que les enquêtés ont de leur décision d'être dirigeant créateur. Il s'agit de caractériser ces grandes tendances selon les 8 éléments d'identification.

Afin de pouvoir identifier au mieux les diverses perceptions des entrepreneurs, nous avons créé un tableau numérique de 172 lignes correspondant aux entrepreneurs enquêtés et de 113 colonnes dont 8 correspondent aux éléments d'identification et les 95 restantes aux questions non agrégées. Ensuite, nous avons soumis ce tableau numérique aux techniques statistiques d'analyse de données dite factorielle (Analyse des Correspondances 
Multiples) complétées par une technique de classification hiérarchique ascendante pour séparer et regrouper les entrepreneurs en différentes classes. Ces méthodes sont destinées à fournir des représentations graphiques simples et des groupes plus ou moins homogènes. Nous avons utilisé les logiciels statistiques SPAD_5.0 et SPSS 10.0.

Afin d'identifier au mieux les entrepreneurs, nous nous sommes focalisés dans un premier temps sur les 8 éléments d'identification des entrepreneurs et les 95 variables non agrégées (questions).

Pour comprendre au mieux les entrepreneurs enquêtés, des tendances doivent être dégagées de l'ensemble des données à analyser c'est-à-dire que dans un premier temps, il s'agit de rechercher un ou plusieurs axes principaux (tendances) pour pouvoir classer et établir la typologie des entrepreneurs en plusieurs groupes de mêmes caractéristiques d'identification et de perceptions du métier de dirigeant de PME au sens du potentiel entrepreneurial. Ces tendances sont générées (en utilisant le logiciel SPAD_5.0) et consignées dans le tableau $\mathrm{n}^{\circ} 1$.

Tableau 1. Pourcentages des quatre premières valeurs propres

\begin{tabular}{|l|c|c|c|}
\hline Numéro & $\begin{array}{c}\text { Valeur } \\
\text { propre }\end{array}$ & Pourcentage & $\begin{array}{c}\text { Pourcentage } \\
\text { cumulé }\end{array}$ \\
\hline 1 & 0,2005 & 7,24 & 7,24 \\
\hline 2 & 0,0962 & 3,48 & 10,72 \\
\hline 3 & 0,0579 & 2,09 & 12,81 \\
\hline 4 & 0,0553 & 2,00 & 14,81 \\
\hline
\end{tabular}

Les deux premières valeurs propres recueillent à elles seules $10,72 \%$ d'information. Elles correspondent aux deux tendances les plus importantes. Les autres valeurs propres ne contiennent qu'un faible pourcentage d'information comparée aux deux premières et n'apportent guère d'éléments significatifs supplémentaires. Afin de mener à bien cette étude, nous étudierons les deux premières tendances.

\subsection{Description de la première tendance : domination des facteurs de motivation}

La première tendance correspond au premier axe factoriel. Cet axe factoriel a un taux d'inertie de 7,24 \% c'est-à-dire que cette première tendance regroupe 7,24\% d'information'.

\footnotetext{
${ }^{4}$ La faiblesse de ces informations rendues est normale et caractéristique de la technique ACM (Analyse en Composantes Multiples) qui opère généralement sur des tableaux de grandes tailles avec de faibles valeurs.
} 
Tableau 2. Première tendance des caractéristiques entrepreneuriales significativement perçues

\begin{tabular}{|c|c|c|c|c|}
\hline $\begin{array}{l}\text { Libellé des } \\
\text { caractéristiques } \\
\text { entrepreneuriales }\end{array}$ & $\begin{array}{l}\text { Libellé de la } \\
\text { modalité }\end{array}$ & $\begin{array}{c}\text { Pourcentage } \\
\text { de la } \\
\text { question } \\
\text { dans l'axe }\end{array}$ & $\begin{array}{c}\text { Pourcentage de } \\
\text { la } \\
\text { caractéristique } \\
\text { dans la } \\
\text { dimension }\end{array}$ & $\begin{array}{l}\text { Dimension clé à } \\
\text { laquelle se } \\
\text { rapporte la } \\
\text { caractéristique }\end{array}$ \\
\hline $\begin{array}{l}\text { A l'aise pour prendre des } \\
\text { décisions autonomes }\end{array}$ & $\begin{array}{l}\text { Totalement } \\
\text { en accord }\end{array}$ & 2,1 & 59,52 & Autonomie \\
\hline $\begin{array}{c}\text { Volonté d'aller jusqu'au } \\
\text { bout de la tâche à } \\
\text { accomplir peu importe les } \\
\text { obstacles }\end{array}$ & $\begin{array}{l}\text { Totalement } \\
\text { en accord }\end{array}$ & 2,04 & 66,18 & Persévérance \\
\hline $\begin{array}{c}\text { Volonté absolue à fixer ses } \\
\text { propres objectifs }\end{array}$ & $\begin{array}{l}\text { Totalement } \\
\text { en accord }\end{array}$ & 1,89 & 61,38 & Accomplissement \\
\hline $\begin{array}{l}\text { Aisance au niveau du } \\
\text { travail nécessitant des } \\
\text { décisions rapides }\end{array}$ & $\begin{array}{l}\text { Totalement } \\
\text { en accord }\end{array}$ & 1,86 & 67,74 & $\begin{array}{l}\text { Coaction } \\
\text { (conception et } \\
\text { réalisation de } \\
\text { projet) }\end{array}$ \\
\hline $\begin{array}{l}\text { Volonté absolue à mener à } \\
\text { terme les projets exigeants }\end{array}$ & $\begin{array}{l}\text { Totalement } \\
\text { en accord }\end{array}$ & 1,82 & 44,51 & Accomplissement \\
\hline $\begin{array}{l}\text { Etre meilleur que les autres } \\
\text { dans son domaine }\end{array}$ & $\begin{array}{l}\text { Totalement } \\
\text { en accord }\end{array}$ & 1,6 & 53,75 & $\begin{array}{l}\text { Accomplissement } \\
\text { et Pouvoir }\end{array}$ \\
\hline $\begin{array}{l}\text { Aisance à accomplir } \\
\text { plusieurs tâches }\end{array}$ & $\begin{array}{l}\text { Totalement } \\
\text { en accord }\end{array}$ & 1,57 & 42,68 & Energie et Stress \\
\hline $\begin{array}{l}\text { Bonne marge de manœuvre } \\
\text { comme source d'efficacité }\end{array}$ & $\begin{array}{l}\text { Totalement } \\
\text { en accord }\end{array}$ & 1,56 & 58,97 & Autonomie \\
\hline
\end{tabular}

Cette première tendance est caractérisée essentiellement par le total accord des entrepreneurs au niveau de sept dimensions clés: autonomie, persévérance, accomplissement, coaction, pouvoir, énergie et tolérance au stress. Ces sept dimensions cumulent $14.44 \%$ de l'information sur ce premier axe factoriel. On peut les articuler autour de trois types de variables.

- Variables de motivation: accomplissement, autonomie et pouvoir, avec plus de $62 \%$ de l'information ( $8.97 \%$ sur $14.44 \%$ );

- Variables d'aptitude au sens de capacité professionnelle: énergie, persévérance et stress, avec $25 \%$ de l'information (3.61\% sur 14.44\%);

- Variables d'action: coaction (conception et réalisation de projet), avec près de $13 \%$ de l'information ( $1.86 \%$ sur $14.44 \%$ ).

Nous observons que l'accomplissement est une variable fréquente dans cette première tendance (près de 37\% de l'information. S'y ajoute l'autonomie (plus de $25 \%$ de l'information). Autrement dit, les variables de motivation dominent ce premier axe factoriel. 
4.2 Description de la deuxième tendance : prééminence des facteurs d'aptitude

La deuxième tendance correspond au deuxième axe factoriel. Cet axe regroupe $3,48 \%$ de l'information.

Tableau 3. Deuxième tendance des caractéristiques entrepreneuriales significativement perçues

\begin{tabular}{|c|c|c|c|c|}
\hline $\begin{array}{l}\text { Libellé des caractéristiques } \\
\text { entrepreneuriales }\end{array}$ & $\begin{array}{l}\text { Libellé de la } \\
\text { modalité }\end{array}$ & $\begin{array}{l}\text { Contribution } \\
\text { de la question } \\
\text { sur l'axe }\end{array}$ & $\begin{array}{c}\text { Pourcentage } \\
\text { de la } \\
\text { caractéristiqu } \\
\text { e dans la } \\
\text { dimension }\end{array}$ & $\begin{array}{l}\text { Dimension clé à } \\
\text { laquelle se } \\
\text { rapporte la } \\
\text { caractéristique }\end{array}$ \\
\hline $\begin{array}{l}\text { Concurrence comme source } \\
\text { d'efficacité }\end{array}$ & $\begin{array}{l}\text { Totalement } \\
\text { en désaccord }\end{array}$ & 3,48 & 55,46 & Concurrence \\
\hline $\begin{array}{l}\text { Facilité d'adaptation face à } \\
\text { des changements importants }\end{array}$ & $\begin{array}{l}\text { Plutôt en } \\
\text { désaccord }\end{array}$ & 3,39 & 39,82 & Changement \\
\hline $\begin{array}{c}\text { Concurrence comme source } \\
\text { de stimulation compétitive } \\
\text { dans les affaires }\end{array}$ & $\begin{array}{l}\text { Totalement } \\
\text { en désaccord }\end{array}$ & 2,91 & 80,41 & Concurrence \\
\hline $\begin{array}{l}\text { Toutes circonstances comme } \\
\text { source de profit }\end{array}$ & $\begin{array}{l}\text { Plutôt en } \\
\text { désaccord }\end{array}$ & 2,9 & 61,72 & $\begin{array}{l}\text { Coaction } \\
\text { (conception et } \\
\text { réalisation de } \\
\text { projet) }\end{array}$ \\
\hline $\begin{array}{l}\text { Volonté de connaître les } \\
\text { nouveautés dans mon champ } \\
\text { d'activité }\end{array}$ & $\begin{array}{l}\text { Plutôt en } \\
\text { désaccord }\end{array}$ & 2,62 & 43,13 & Cognition \\
\hline $\begin{array}{l}\text { Préférence pour les travaux à } \\
\text { fortes responsabilités }\end{array}$ & $\begin{array}{l}\text { Totalement } \\
\text { en désaccord }\end{array}$ & 2,53 & 61,66 & Pouvoir \\
\hline $\begin{array}{c}\text { Aisance dans les discussions } \\
\text { avec des dirigeants même en } \\
\text { cas de désaccord }\end{array}$ & $\begin{array}{l}\text { Totalement } \\
\text { en désaccord }\end{array}$ & 2,46 & 64,23 & $\begin{array}{l}\text { Confiance en soi } \\
\text { et Affectivité }\end{array}$ \\
\hline $\begin{array}{l}\text { Imagination de multiples } \\
\text { utilisations à des objets } \\
\text { d'usage courant }\end{array}$ & $\begin{array}{c}\text { Totalement } \\
\text { en désaccord }\end{array}$ & 2,44 & 52,05 & Innovation \\
\hline $\begin{array}{l}\text { Volonté d'aller jusqu'au bout } \\
\text { de ce qui est commencé peu } \\
\text { importent les difficultés }\end{array}$ & $\begin{array}{l}\text { Totalement } \\
\text { en désaccord }\end{array}$ & 2,41 & 71,37 & Persévérance \\
\hline
\end{tabular}

Cette deuxième tendance met en évidence les attitudes face à la concurrence et au changement, le comportement dans l'action, la cognition, le pouvoir, la confiance en soi, le comportement affectif, l'innovation et la persévérance.

Contrairement à la première tendance, les entrepreneurs associés à cette deuxième tendance sont totalement ou plutôt en désaccord avec certaines idées proposées. Nous les regroupons en trois catégories de variables : 
- Variables d'aptitude au sens de capacité professionnelle: capacité de différenciation (attitude face au changement et à la concurrence, et intérêt pour l'innovation), confiance en soi, affectivité et persévérance;

- Variables de motivation: pouvoir;

- Variables d'action: coaction (conception et réalisation de projet) et cognition.

Nous observons que les variables d'aptitude caractérisent mieux cette deuxième tendance. Elles représentent près de $70 \%$ (17.09\% sur $25.14 \%)$ de l'information que l'ensemble des caractéristiques entrepreneuriales significativement perçues concentre sur le deuxième axe factoriel. La prise en compte de la concurrence y est fortement représentée, avec plus du quart de cette même information soit $6.39 \%(3.48+2.91)$ sur $25.14 \%$.

Le contenu de ces deux premières tendances fondamentales montre que le comportement entrepreneurial du dirigeant de PME résulte des variables de motivation et d'aptitude. Les résultats fournis par l'analyse des correspondances multiples sont significatifs. En fait, cette technique permet de partir des énoncés en les considérant comme des variables à par entière. Des recombinaisons sont effectuées et réorientées vers les dimensions. Même s'il s'agit d'une technique exploratoire, elle a le mérite de réduire le nombre d'énoncés et de résumer les relations entre les variables à l'aide d'un ensemble de facteurs aussi restreint que possible qui est censé discriminer les données de manière à être facilement interprétable. Elle est complétée par la classification ascendante hiérarchique qui s'interprète en termes de partitions. Une partition n'est intéressante que si les classes sont nettement individualisées, c'est-à-dire qu'elles doivent former chacune un tout cohérent, bien caractérisé (compacité) et être distinctes les unes des autres (séparabilité). C'est en vertu de ces considérations méthodologiques que nous retenons les quatre classes les plus stables. La prise en compte de leur contenu nous autorise à proposer une typologie de trois groupes d'entrepreneurs en termes de comportements décisionnels.

\section{DIFFERENCIATION DES COMPORTEMENTS DECISIONNELS ISSUE DES CARACTERISTIQUES ENTREPRENEURIALES}

Après avoir déterminé les deux grandes tendances de ces entrepreneurs, il s'agit ici de les regrouper et d'établir une typologie de classes d'entrepreneurs par éléments d'identification et par variables clés traduisant leurs comportements décisionnels. Afin de mener à bien cette étude, ce traitement de données a été effectué à l'aide d'une Analyse des Correspondances Multiples suivie d'une Classification Ascendante Hiérarchique sur le logiciel SPAD_5.0. Quatre classes résultent de cette analyse et se positionnent comme suivant sur le graphique des deux grandes tendances réalisé précédemment. 
Graphique 1 - Projection plane des quatre classes d'entrepreneurs

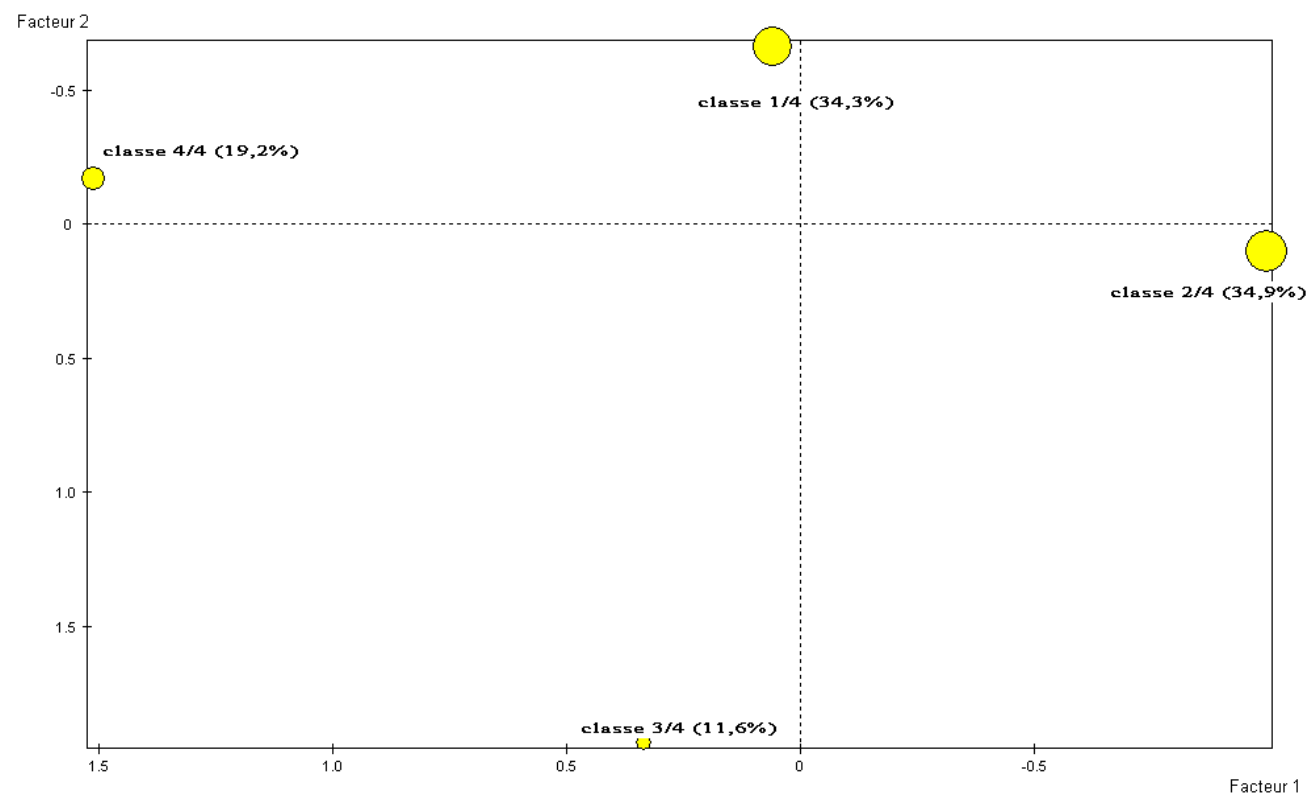

Ces quatre classes bien distinctes suivent les tendances étudiées précédemment (facteurs 1 et 2). En effet, la classe 1/4 (59 personnes) est opposée à la classe 3/4 (20 personnes) sur le premier axe factoriel et la classe 2/4 (60 personnes) à la classe 4/4 (33 personnes) sur le deuxième axe factoriel. L'étude de ces classes équivaut à rechercher des profils d'entrepreneurs décideurs plus ou moins complets. Afin de compléter ces premières tendances et de regrouper au mieux les entrepreneurs enquêtés, nous avons effectué une classification hiérarchique ascendante sur les deux facteurs étudiés précédemment (axes).

Graphique 2 - Arborescence selon l'histoire de vie et les perceptions des entrepreneurs

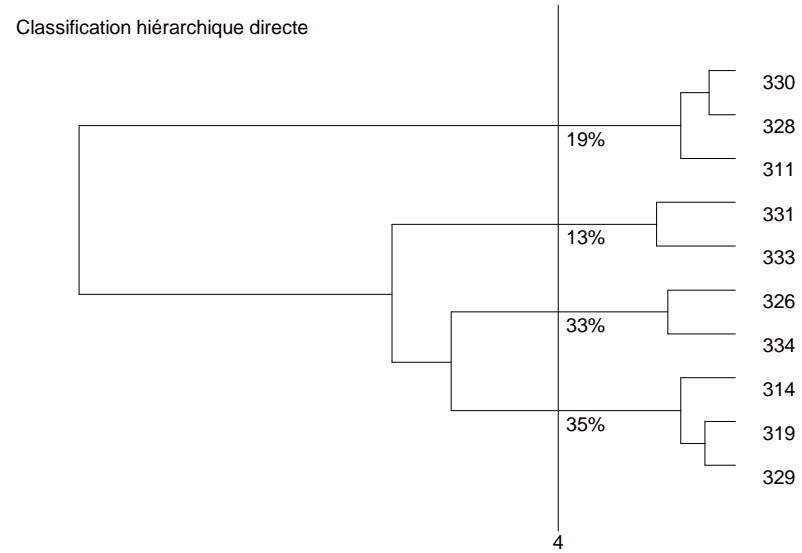


Cet arbre montre le mode de rattachement des entrepreneurs les uns aux autres. Nous ne présentons ici que les dix derniers nœuds autrement dit les branches supérieures car nous étudions quatre classes. Nous remarquons l'existence d'une classe relativement stable comprenant $19 \%$ des entrepreneurs c'est-à-dire qu'elle se rattache aux autres branches de l'arbre à son sommet. Cette classe correspond à la classe 4/4. De plus, un tel constat suppose une certaine stabilité des réponses des entrepreneurs composant cette classe. Nous retrouvons également les trois autres classes 1/4, 2/4 et 3/4. Par ailleurs, l'Analyse des Correspondances Multiples détermine les questions ainsi que les modalités associées qui caractérisent au mieux les classes. Cette analyse fournit des contenus identiques pour les clases $1 / 4$ et 2/4. Nous pouvons ainsi les agréger dans un seul groupe d'entrepreneurs. En définitive, nous obtenons trois catégories d'entrepreneurs au vu de leurs opinions sur les caractéristiques déterminant leurs comportements décisionnels.

\subsection{Les entrepreneurs de décisions non courantes - $68 \%$ de l'effectif enquêté}

Ils sont issus de l'addition des classes $1 / 4$ et $2 / 4$ compte tenu de la quasi-concordance au niveau de leurs caractéristiques entrepreneuriales. Ils s'apparentent aux créateurs gestionnaires de Baumol (1968) et sont au cœur d'un construit systémique et socialement organisé. Leurs caractéristiques semblent être liées à un comportement plus actif vis-à-vis de l'environnement de leur entreprise.

Tableau 4. Principales caractéristiques entrepreneuriales (classe $1 / 4$ et classe 2/4)

\begin{tabular}{|c|l|c|c|l|}
\hline $\begin{array}{c}\text { Libellés des caractéristiques } \\
\text { entrepreneuriales }\end{array}$ & $\begin{array}{c}\text { Libellés } \\
\text { des } \\
\text { modalités }\end{array}$ & $\begin{array}{c}\text { Poids (\%) de la } \\
\text { caractéristique } \\
\text { dans l'échantillon }\end{array}$ & $\begin{array}{c}\text { Poids (\%) de } \\
\text { la } \\
\text { caractéristiq } \\
\text { ue dans le } \\
\text { groupe }\end{array}$ & $\begin{array}{c}\text { Dimension } \\
\text { clé à laquelle } \\
\text { se rattache la } \\
\text { caractéristiq } \\
\text { ue }\end{array}$ \\
\hline $\begin{array}{c}\text { Volonté de connaître les } \\
\text { nouveautés dans mon champ } \\
\text { d'activité }\end{array}$ & $\begin{array}{l}\text { Totalement } \\
\text { en accord }\end{array}$ & 47.10 & 69.50 & Cognition \\
\hline $\begin{array}{c}\text { Capacité à créer des changements } \\
\text { organisationnels comme source } \\
\text { de réussite de l'entrepreneur }\end{array}$ & $\begin{array}{l}\text { Plutôt en } \\
\text { accord }\end{array}$ & 46.51 & 75.00 & Changement \\
\hline $\begin{array}{c}\text { Volonté absolue à fixer ses } \\
\text { propres objectifs }\end{array}$ & $\begin{array}{l}\text { Plutôt en } \\
\text { accord }\end{array}$ & 57.56 & 85.00 & $\begin{array}{l}\text { Accomplisse } \\
\text { ment }\end{array}$ \\
\hline $\begin{array}{c}\text { Toujours plus productif avec un } \\
\text { niveau de stress élevé }\end{array}$ & $\begin{array}{l}\text { Plutôt en } \\
\text { désaccord }\end{array}$ & 41.86 & 70.00 & Stress \\
\hline $\begin{array}{c}\text { Importance de suivre les projets } \\
\text { de leur début jusqu'à leur fin }\end{array}$ & $\begin{array}{l}\text { Plutôt en } \\
\text { accord }\end{array}$ & 44.19 & 71.67 & $\begin{array}{l}\text { Innovation et } \\
\text { Changement }\end{array}$ \\
\hline $\begin{array}{c}\text { A l'aise pour prendre des } \\
\text { décisions autonomes }\end{array}$ & $\begin{array}{l}\text { Plutôt en } \\
\text { accord }\end{array}$ & 53.49 & 80.00 & Autonomie \\
\hline $\begin{array}{c}\text { Concurrence comme source } \\
\text { d'efficacité }\end{array}$ & $\begin{array}{l}\text { Plutôt en } \\
\text { accord }\end{array}$ & 40.12 & 66.67 & Concurrence \\
\hline $\begin{array}{c}\text { Volonté de mener jusqu'au bout } \\
\text { un projet entrepris même difficile }\end{array}$ & $\begin{array}{l}\text { Plutôt en } \\
\text { accord }\end{array}$ & 39.53 & 65.00 & Persévérance \\
\hline Enclin à être patron & $\begin{array}{l}\text { Plutôt en } \\
\text { accord }\end{array}$ & 41.28 & 66.67 & Pouvoir \\
\hline $\begin{array}{c}\text { Résultats obtenus comme source } \\
\text { d'évaluation des progrès } \\
\text { personnels }\end{array}$ & $\begin{array}{l}\text { Plutôt en } \\
\text { accord }\end{array}$ & 48.84 & 73.33 & Cognition \\
\hline $\begin{array}{l}\text { Volonté d'aller jusqu'au bout de ce } \\
\text { qui est commencé peu importe les } \\
\text { difficultés }\end{array}$ & $\begin{array}{l}\text { Plutôt en } \\
\text { accord }\end{array}$ & 50.58 & 75.00 & Persévérance \\
\hline
\end{tabular}


Les entrepreneurs de la première sous classe ne sont pas identifiables par leur sexe car à $1 \%$ près, les effectifs d'hommes et de femmes de la classe $1 / 4$ et de l'échantillon sont égaux. Leur âge est significatif. En moyenne, ces entrepreneurs sont plus âgés que ceux de l'échantillon. En effet, la différence de pourcentage est de $11 \%$ pour les personnes de plus de 40 ans. De même, ces entrepreneurs semblent être plus diplômés que ceux de l'échantillon $(\mathrm{Bac}+2$ à $\mathrm{Bac}+3$ ou plus). Ces entrepreneurs ont tendance à être propriétaires de leur entreprise. Leurs parents n'ont pas exercé d'activité à leur propre compte et n'ont jamais possédé d'entreprise. De plus, ils n'ont pas plus d'expérience professionnelle dans une PME que les entrepreneurs de l'échantillon ni d'expérience multiple de créateur d'entreprise. Enfin, ils accordent deux fois plus d'importance aux variables d'aptitude par rapport aux facteurs de motivation. Mais ils se différencient des autres par leur forte motivation à acquérir de nouvelles connaissances (cognition) au regard de leur contexte d'action. En fait, certaines composantes des trois dimensions clés de la motivation (accomplissement, autonomie et pouvoir) sont corrélées avec la cognition.

Dans la deuxième sous classe, les hommes ont une très légère tendance à être plus nombreux que dans l'échantillon. Leur âge semble se situer plus particulièrement entre 35 et 45 ans tandis qu'ils ont une scolarité de niveau BEPC ou de niveau Bac. Ces dirigeants d'entreprise ont une culture d'indépendant du fait des parents entrepreneurs et/ou d'avoir eux-mêmes l'expérience antérieure de création d'entreprise. Ils sont $6 \%$ de plus à avoir créé une entreprise de très petite ou de petite taille que ceux de l'échantillon. Ils en sont souvent à leur deuxième expérience. Souvent, ils se refusent de s'arrêter sur des schémas acquis et tentent de nouveaux modèles afin d'aller au-delà des choix routiniers.

\section{- Une prééminence des décisions non routières}

Les analyses modernes de l'entrepreneur développent la vision de Say en disant que l'organisation, la production et la combinaison des apports de ressources nécessitent des compétences d'un autre ordre que ceux du travail de routine. Des chercheurs tels que Schumpeter (1936; 1942), Knight (1921) et Kirzner (1983) fournissent une caractérisation de l'entrepreneur de telle sorte que la fonction d'entreprise appartient à la sphère de la prise de décision «non-routinière »: les décisions d'entreprise ne sont pas suffisamment semblables, et ne reviennent pas assez souvent, pour justifier l'élaboration de procédures de routine. L'absence d'un rôle actif de l'entrepreneur dans l'économie néoclassique dérive directement sur l'hypothèse de l'équilibre du marché: des changements peuvent se produire, mais les variations ont été parfaitement anticipées et les attentes ne pourront jamais être déçues. Selon Knight (1921), cependant, le changement ne donne pas lieu à des bénéfices, mais l'incertitude et la possibilité d'inexactitude des attentes oui.

L'incertitude souligne la volonté de l'entrepreneur et sa capacité à assumer la responsabilité de la prise de décision: en particulier, «l'incertitude de Knight" se réfère à une situation où il n'y a pas d'expérience factuelle pour soutenir la fixation de probabilités objectives à des évènements pertinents (par rapport au cas de risque, lorsque les probabilités peuvent être attribuées). Si c'est l'incertitude qui caractérise l'environnement, le problème n'est plus représenté par l'exécution effective de l'activité: la question est de savoir quoi faire et comment le faire. Cette «fonction principale» est l'essence même de l'esprit d'entreprise: l'effort de décider comment les différents objectifs seront atteints et de prévoir que les objectifs soient réalisés par l'entrepreneur, "le spécialiste prêt à supporter 
le coût de l'incertitude» (Knight, 1921). Les théories récentes, en particulier, soulignent que l'entrepreneur ne se contente pas de faire face à la conséquence de la connaissance imparfaite, mais vise plutôt la récompense de la découverte et l'utilisation de nouvelles connaissances. La décision d'entreprise repose sur le génie, la personnalité et le talent d'innovateur-entrepreneur avec le souci permanent d'un comportement actif vis-à-vis des contextes interne et externe.

\section{- Permanence d'un construit systémique et socialement organisé}

La conception décisionnelle du dirigeant créateur non routinier serait l'œuvre d'une construction cognitive complexe. Autrement dit, la représentation qu'il se fait de ses caractéristiques entrepreneuriales résultent de ses expériences sensibles et cognitives avec une certaine pertinente que celle déduite par l'esprit rationnel et précis. Ce qui veut dire aussi qu'une telle représentation réintroduit l'entrepreneur dans l'acte de construction de la connaissance (Le Moigne , 1995, p. 97). En réalité, nous pensons que les interactions entre le cheminement de pensée et de la praxis, entre la réflexion et l'action, donnent un sens aux activités, aux conduites et aux comportements des entrepreneurs. Elles conditionnent d'une façon dynamique non linéaire (au fil du temps, des actions et des résultats obtenus) leurs structures et leurs processus cognitifs et organisationnels (Stacey, 1995) sous-tendus par les caractéristiques entrepreneuriales évoquées.

C'est dire qu'au niveau de la création d'entreprise, nous admettons que des intentionnalités multiples et diverses engagées dans un flux d'actions s'appuient sur des expériences sensibles et des connaissances fonctionnelles tangibles et intelligibles (Raux, 1995 p. 210 et s.) qui, à leur tour, confirment ou altèrent les structures et les processus cognitifs des sujets pensants et agissants qui animent l'organisation créée et ponctuent sa dynamique. C'est par cette dialectique entre Empirie et Epistémé (Le Moigne, 1995 p. 104) ou encore entre praxis et conceptulis ${ }^{5}$, que les dirigeants créateurs non routiniers se sentent guidés à la fois par un savoir-faire, une connaissance pertinente préalable à l'action. Ceci est dû au fait qu'ils apprennent consciemment ou tacitement dans et de l'action elle-même. D'où cet appel insistant de Michel CROZIER (1989, pp. 48-50) à réconcilier les deux dimensions de la « réalité » des systèmes sociaux :

«Les problèmes d'organisations sont à la fois conceptuels et existentiels. Le vécu précède l'idée, mais seule l'idée permet de comprendre le vécu et donc de le formaliser, de le développer et de reculer des limites du possible (p. 49)... Les entreprises ne font pas émerger de nouveaux principes par génération spontanée. Elles ont besoin de la stimulation qu'apporte l'introduction d'un raisonnement formalisé. Mais ce raisonnement formalisé n'a pas de chance de réussir s'il est imposé à partir d'une réflexion technocratique détachée de l'expérience (p. 50)»

\subsection{Les entrepreneurs de décisions de routine - $13 \%$ de l'effectif enquêté}

Ils semblent s'éloigner à la fois des créateurs gestionnaires et des créateurs entrepreneurs de Baumol (1968). Ils sont au cœur d'un construit contextuel fort mais limité aux activités quotidiennes. Leurs caractéristiques semblent être liées à un comportement plus passif vis-à-vis de l'environnement de leur entreprise.

${ }^{5}$ On lira quelques-uns des travaux de Abraham A. MOLES, notamment «Les sciences de l'imprécis » Édition du Seuil, série, Science ouverte, Paris, 1990, «Théories des actes. Vers une écologie des actions » Casterman, Paris, 1977 ou encore «Labyrinthes du vécu » Klincksieck, Paris, 1982. 
Tableau 5. Principales caractéristiques entrepreneuriales (classe 3/4)

\begin{tabular}{|c|l|c|c|l|}
\hline $\begin{array}{c}\text { Libellés des caractéristiques } \\
\text { entrepreneuriales }\end{array}$ & $\begin{array}{c}\text { Libellés des } \\
\text { modalités }\end{array}$ & $\begin{array}{c}\text { Poids (\%) } \\
\text { de la } \\
\text { caractéristiq } \\
\text { ue dans } \\
\text { l'échantillon }\end{array}$ & $\begin{array}{c}\text { Poids (\%) } \\
\text { de la } \\
\text { caractéristiq } \\
\text { ue dans le } \\
\text { groupe }\end{array}$ & $\begin{array}{c}\text { Dimension clé à } \\
\text { laquelle se } \\
\text { rattache la } \\
\text { caractéristique }\end{array}$ \\
\hline $\begin{array}{c}\text { Premier à essayer les } \\
\text { nouveaux produits qui sortent } \\
\text { sur le marché }\end{array}$ & $\begin{array}{l}\text { Totalement en } \\
\text { désaccord }\end{array}$ & 10.47 & 50.00 & Innovation \\
\hline $\begin{array}{c}\text { Vision toujours positive des } \\
\text { choses }\end{array}$ & $\begin{array}{l}\text { Totalement en } \\
\text { désaccord }\end{array}$ & 3.49 & 25.00 & Stress \\
\hline $\begin{array}{c}\text { Volonté absolue à accomplir } \\
\text { les choses difficiles }\end{array}$ & $\begin{array}{l}\text { Totalement en } \\
\text { désaccord }\end{array}$ & 9.88 & 40.00 & $\begin{array}{l}\text { Accomplissemen } \\
\text { t }\end{array}$ \\
\hline $\begin{array}{c}\text { Volonté d'aller jusqu'au bout } \\
\text { de ce qui est commencé peu } \\
\text { importe les difficultés }\end{array}$ & $\begin{array}{l}\text { Totalement en } \\
\text { désaccord }\end{array}$ & 2.33 & 20.00 & Persévérance \\
\hline $\begin{array}{c}\text { Aisance dans les discussions } \\
\text { avec des dirigeants même en } \\
\text { cas de désaccord }\end{array}$ & $\begin{array}{l}\text { Totalement en } \\
\text { désaccord }\end{array}$ & 2.33 & 20.00 & $\begin{array}{l}\text { Confiance en soi } \\
\text { et Affectivité }\end{array}$ \\
\hline $\begin{array}{c}\text { Concurrence comme source } \\
\text { de stimulation compétitive } \\
\text { dans les affaires }\end{array}$ & $\begin{array}{l}\text { Totalement en } \\
\text { désaccord }\end{array}$ & 4.07 & 25.00 & Concurrence \\
\hline $\begin{array}{c}\text { Aisance à influencer les } \\
\text { autres et à les conduire à faire } \\
\text { ce que je veux qu'ils fassent }\end{array}$ & $\begin{array}{l}\text { Totalement en } \\
\text { accord }\end{array}$ & 17.44 & 50.00 & Cognition \\
\hline
\end{tabular}

Les questions de modalité 1 (totalement en désaccord) caractérisent fondamentalement ce troisième groupe. Même si cette perception du potentiel entrepreneurial du dirigeant de PME n'est pas grandement affirmée, les avis des entrepreneurs de cette classe convergent sur la modalité totalement en désaccord : ces entrepreneurs semblent évaluer de façon faible les dimensions de motivation et d'aptitude relative à leur potentiel de dirigeant. Parmi ces dirigeants, les femmes sont fortement représentées avec une différence de 19\% par rapport à l'ensemble de l'échantillon. Ils ont significativement entre 30 et 40 ans voire entre 20 et 40 ans. Leur niveau scolaire se situe à celui du BEPC ou quelque fois au Bac +2 ; ceci laisse à penser que les entrepreneurs les plus âgés sont diplômés d'un BEPC tandis que les plus jeunes ont $\mathrm{Bac}+2$. Le pourcentage de travailleurs à leur compte est également significatif. Très peu ont eu d'expérience professionnelle dans une PME avant leur acte de création d'entreprise. Cet élément d'identification est très nettement caractéristique de ce groupe au vu de la grande différence avec l'ensemble de l'échantillon. De même, cette classe a tendance à ne pas avoir eu d'expérience antérieure de créateur d'entreprise. Ils sont à leur première création, au sens de profession libérale (travailleur à son compte) ou de micro entreprise. Ils ont tendance à se limiter aux modèles connus et déjà expérimentés avec le souci permanent de mieux coordonner les ressources et moyens existants.

\section{- Une prééminence du rôle de coordinateur}

Dans les modèles traditionnels de l'entreprise, l'entrepreneur a le rôle de coordinateur dans une situation où les ressources sont rares. La coordination peut être analysée d'un point de vue général ou partiel. Des études sur l'équilibre général montrent des modèles purement 
théoriques de marchés qui fonctionnent comme des instruments d'allocation des ressources. Dans ce contexte, le système économique est donc caractérisé par un savoir parfait et les entreprises sont considérées comme des boîtes noires, comme par exemple la transformation d'investissements en production. Lorsque le rôle des entrepreneurs est une simple fonction de coordination des ressources, il reste peu de place pour une théorie de l'esprit d'entreprise: en gros, l'équilibre de l'existence d'un esprit d'entreprise n'est pas nécessaire, étant donné que toutes les ressources sont organisées et affectées de manière efficace (Barreto, 1989). Dans cette ligne de pensée, Leibenstein (1966, 1979) dans sa théorie X-efficacité souligne l'organisation de la fonction de l'entrepreneur. L'entrepreneur qui réussit est celui qui parvient à minimiser les inefficacités qui surgissent inévitablement dans le processus de coordination des facteurs de productions. Le rôle de l'entrepreneur est donc d'améliorer l'efficacité de l'information des flux dans ces marchés, et à «combler l'écart», qui est étroitement apparenté à la fonction d'arbitrage. Ce type d'approche suppose implicitement que la différence entre l'arbitrage simple et la mise en place d'une entreprise réside dans le degré de complexité et le type de contrats en cause plutôt que dans une différence de développement économique. Dans les deux cas, les ressources sont réaffectées, et si cette réaffectation détermine des avantages pour tous, «l'entrepreneur peut faire un grand bénéfice pour son entreprise» (Ricketts, 2002).

L'idée que les gens commencent de nouvelles entreprises comme un moyen d'augmenter leur richesse personnelle sous-tend ce volet de recherche en entrepreneuriat: l'entrepreneur coordonne seulement des ressources, et la possibilité d'allouer ces ressources de façon optimale représente le seul déterminant de la décision entrepreneuriale. La représentation néo-classique de l'entrepreneur se réfère à une personne "qui maximise l'objet du profit de diverses manières " (Day et Sunder, 1996). Des facteurs tels que les revenus de l'entreprise, la rentabilité, la création de richesses personnelles, la croissance et la durabilité sont utilisées comme indicateurs de la réussite entrepreneuriale (Amit et al., 2000), et les critères qui guident la décision entrepreneuriale dans la littérature sont essentiellement la maximisation des profits attendus, l'utilité espérée de maximisation du profit, et la maximisation des entreprises en valeur boursière (Kihlstrom et Laffont, 1979).

\section{- Importance du contexte dans la construction de la décision}

Une réelle prudence émerge des représentations que les dirigeants créateurs routiniers se font de leurs caractéristiques entrepreneuriales. Tout laisse à penser qu'ils sont très sensibles aux oscillations de leurs marchés, à l'écoute qu'ils prêtent aux partenaires et adversaires internes et externes, aux crises et aux événements que traversent leurs organisations (Crozier M., 1989, p. 48), au temps et aux efforts qu'ils aménagent pour répondre aux besoins stratégiques de celles-ci (Eisenhardt K. M., 1992), aux faiblesses et aux réussites qu'ils décèlent intuitivement ou analytiquement dans la stratégie des concurrents (Peters T. et R. Waterman, 1983). Ainsi, ils font le choix d'apprendre peu à peu à accommoder leurs capacités cognitives et leurs représentations afin d'agir efficacement face à la volatilité intrinsèque des environnements incertains et en évolution rapide (Simon H. A. 1993, 134). Ils acceptent de "stabiliser" leur connaissance et apprennent à s'apercevoir des lacunes qui les empêchent de se réaliser chaque fois qu'ils agissent ou qu'ils réfléchissent sur une situation qu'ils perçoivent comme étant significative.

En effet, l'action concrète s'accompagne d'une emprise cognitive confirmative, correctrice, révélatrice et apprenante à la fois. Mais de telles situations deviennent 
rapidement fréquentes et familières qu'elles ne suscitent plus suffisamment leur curiosité intellectuelle. Il s'agit de situations qui structurent la vision et la compréhension du monde qui nous entoure et mettent notre connaissance théorique et notre vécu pratique en étroite symbiose. Pire, elles peuvent nous conduire aux formules standards et aux modèles préétablis dont nous savons pertinemment l'inefficacité face à la singularité des situations concrètes et à la proactivité face aux faits entrepreneuriaux.

\subsection{Les entrepreneurs proactifs - $19 \%$ de l'effectif enquêté}

Ils s'apparentent aux créateurs entrepreneurs de Baumol (1968). Ils sont au centre d'un construit issu des connaissances constamment renouvelées. Leurs caractéristiques semblent être liées à un comportement plus proactif de l'environnement de leur entreprise

Tableau 6. Principales caractéristiques entrepreneuriales des entreprenants (classe 4/4)

\begin{tabular}{|c|c|c|c|c|}
\hline $\begin{array}{c}\text { Libellés des } \\
\text { caractéristiques } \\
\text { entrepreneuriales }\end{array}$ & $\begin{array}{l}\text { Libellés des } \\
\text { modalités }\end{array}$ & $\begin{array}{l}\text { Poids }(\%) \text { de la } \\
\text { caractéristique } \\
\text { dans } \\
\text { l'échantillon }\end{array}$ & $\begin{array}{l}\text { Poids (\%) } \\
\text { de la } \\
\text { caractéristi } \\
\text { que dans le } \\
\text { groupe }\end{array}$ & $\begin{array}{l}\text { Dimension clé à } \\
\text { laquelle se } \\
\text { rattache la } \\
\text { caractéristique }\end{array}$ \\
\hline $\begin{array}{c}\text { Volonté d'aller jusqu'au } \\
\text { bout de la tâche à } \\
\text { accomplir peu importe les } \\
\text { obstacles }\end{array}$ & $\begin{array}{l}\text { Totalement } \\
\text { en accord }\end{array}$ & 33.14 & 87.88 & Persévérance \\
\hline $\begin{array}{c}\text { Volonté absolue à fixer ses } \\
\text { propres objectifs }\end{array}$ & $\begin{array}{l}\text { Totalement } \\
\text { en accord }\end{array}$ & 37.79 & 87.88 & Accomplissement \\
\hline $\begin{array}{l}\text { Aisance au niveau du } \\
\text { travail nécessitant des } \\
\text { décisions rapides }\end{array}$ & $\begin{array}{l}\text { Totalement } \\
\text { en accord }\end{array}$ & 32.56 & 81.82 & $\begin{array}{l}\text { Coaction } \\
\text { (Conception et } \\
\text { réalisation de } \\
\text { projets) }\end{array}$ \\
\hline $\begin{array}{c}\text { Volonté de participation à } \\
\text { des travaux nécessitant un } \\
\text { ajustement à des } \\
\text { compétences différentes }\end{array}$ & $\begin{array}{l}\text { Totalement } \\
\text { en accord }\end{array}$ & 23.26 & 66.67 & $\begin{array}{l}\text { Capacité } \\
\text { conceptuelle, } \\
\text { Cognition et } \\
\text { Changement }\end{array}$ \\
\hline $\begin{array}{l}\text { Compétition comme } \\
\text { stimulant d'efficacité }\end{array}$ & $\begin{array}{l}\text { Totalement } \\
\text { en accord }\end{array}$ & 33.14 & 78.79 & Concurrence \\
\hline $\begin{array}{l}\text { A l'aise pour prendre des } \\
\text { décisions autonomes }\end{array}$ & $\begin{array}{l}\text { Totalement } \\
\text { en accord }\end{array}$ & 38.95 & 84.85 & Autonomie \\
\hline $\begin{array}{c}\text { Volonté constante de prise } \\
\text { d'initiative }\end{array}$ & $\begin{array}{l}\text { Totalement } \\
\text { en accord }\end{array}$ & 40.12 & 84.85 & $\begin{array}{l}\text { Coaction } \\
\text { (Conception et } \\
\text { réalisation de } \\
\text { projets) }\end{array}$ \\
\hline $\begin{array}{c}\text { Assurance dans } \\
\text { l'expression des opinions }\end{array}$ & $\begin{array}{l}\text { Totalement } \\
\text { en accord }\end{array}$ & 24.42 & 66.67 & Confiance en soi \\
\hline $\begin{array}{l}\text { Préférence pour les travaux } \\
\text { à fortes responsabilités }\end{array}$ & $\begin{array}{l}\text { Totalement } \\
\text { en accord }\end{array}$ & 31.98 & 75.76 & Pouvoir \\
\hline $\begin{array}{c}\text { Importance des } \\
\text { méthodologies d'action } \\
\text { débouchant rapidement sur } \\
\text { des résultats }\end{array}$ & $\begin{array}{l}\text { Totalement } \\
\text { en accord }\end{array}$ & 28.49 & 69.70 & Cognition \\
\hline
\end{tabular}


Cette classe est fortement caractérisée par une réponse de modalité 4 (totalement en accord) aux questions énumérées ci-dessus. Les pourcentages de ces questions sont très largement supérieurs à ceux de l'échantillon, ce qui confirme une perception très convergente des entrepreneurs de cette classe.

Ce sont en grande majorité des hommes de 50 ans et plus, voire entre 25 et 35 ans. Contrairement à toute attente, ils sont de niveau scolaire soit études primaires soit $\mathrm{Bac}+3$ ou plus c'est-à-dire qu'ils sont très bien ou aucunement diplômés.

Ils sont à leur première création d'entreprise (notamment de petite ou de moyenne taille) après une expérience professionnelle en PME. Mais ils ne sont pas issus de milieu familial d'indépendants ou d'entrepreneurs même s'ils semblent affirmer une position très forte des dimensions de motivation et d'aptitude relative à leur potentiel de dirigeant. Ils se différencient complètement des autres groupes de dirigeants à travers une espèce de «permanence d'un construit de décision issu des connaissances constamment renouvelées».

La nature des représentations que les dirigeants créateurs entreprenants se font de leurs caractéristiques entrepreneuriales fait apparaître l'importance du construit dans et par l'action et la réflexion. Un tel construit résulte des représentations tangibles et intelligibles à partir de «leurs expériences sensibles et cognitives» (Le Moigne, 1995, p. 97) soustendues par les caractéristiques entrepreneuriales considérées comme des connaissances utiles à la prise de décision. Le «pragmatisme» et «l'artefact» de Herbert A. SIMON sont deux concepts qui attribuent à la formation d'une connaissance fonctionnelle, celle qui naît dans et des interactions des créateurs entreprenants ou projectifs, un rôle fondamental dans la modélisation du cheminement entrepreneurial.

En considérant le produit des interactions entre les caractéristiques entrepreneuriales des créateurs et les objets de leurs actions dans le processus d'apprentissage, nous prenons en compte les dimensions constructives et créatives de la connaissance que tire l'entrepreneur de son expérience. Ceci dit, nous ne sommes pas loin des propositions factuelles $(f a c t)$ de H. A. SIMON, où les énoncés d'une science se réfèrent «à des faits (ce qui est), à l'expérience, à des événements qui se produisent, ou ne se produisent pas » (Fiol et Sole, 1993, p. 88).

Nous ne réinventons pas l'entrepreneuriat en disant qu'une étude sur le cheminement des entrepreneurs, qui se veut globalement satisfaisante, ne peut se permettre d'ignorer la bipolarité des relations qui lient l'entreprise à son contexte d'action et de réflexion, autrement dit à son environnement. Traditionnellement donc, la notion d'environnement est très importante pour comprendre simultanément la dynamique interne des firmes et leurs comportements externes. Dans un souci de clarté et d'approfondissement de la recherche sur les relations Environnement-Entreprise nous proposons un enrichissement du concept à partir de ses composantes. Du point de vue explicite, l'environnement de l'entrepreneur est fait d'entités distinctes comme: le marché, le secteur économique ou industriel, la branche d'activité, les niveaux hiérarchiques, les ateliers de production, le poste de travail, la société, les institutions gouvernementales, les boutiques de gestion, les organisations syndicales, patronales, professionnelles et éducatives, les organisations concurrentes directes et potentielles, les firmes-clientes, les firmes-fournisseuses, les institutions financières, etc. Au-delà des représentations que l'entrepreneur fait de ces entités, celles-ci constituent des sites symboliques au regard des réalités économiques, sociologiques et culturelles passées, 
actuelles ou pressenties (Dearborn et Simon, 1958; Zaoual, 1996). Il les intègre dans le processus décisionnel compte tenu de ses capacités et aptitudes cognitives et celles propres à son entreprise. A savoir, une extension cognitive, psychologique et sociologique des notions qui le composent : le champ, les entités distinctes, les interactions auxquelles nous ajoutons les délais d'action.

Chaque entité est perçue différemment des autres. Très certainement selon les objectifs de l'observateur (Dearborn et Simon, 1958) mais aussi les comportements de l'organisation observée, ses statuts, ses métiers, ses sites, la composition de son personnel, de son équipe de direction, l'évolution de sa structure, de sa stratégie, de sa production, de son réseau d'influence, etc. Des interactions multiples et diverses entre des entités distinctes se traduiraient par des rapports directs ou indirects d'échange, d'interdépendance et/ou de dépendance; la nature des transactions et la disponibilité ou la rareté des ressources de tout genre y sont déterminantes (Pfeffer et Salancik, 1978; Mintzberg, 1986).

Pratiquement, les créateurs entreprenants seraient plus attentifs aux phénomènes quotidiens suivants: l'asymétrie informationnelle, l'ambiguïté des données, des discours et des comportements organisationnels, l'incertitude des préférences managériales, les choix stratégiques et leurs conséquences. Ils seraient plus conscients du fait que leurs limites cognitives conjuguées à leur partialité de jugement et de décision donneraient des perceptions différentes de "la réalité". Ils seraient plus enclins aux interprétations parfois contradictoires de ce qui paraîtrait semblable dans l'espace et le temps et finiraient par une diversité de comportements élaborés et soutenus face à cette réalité caractérisée par sa complexité et sa variabilité.

\section{CONCLUSION ET PERSPECTIVES DE RECHERCHE}

\section{Eléments de conclusion}

Les résultats de notre étude nous conduisent à trois catégories d'entrepreneurs au vu de leurs opinions sur les caractéristiques déterminant leurs comportements décisionnels. Nous rapprochons ces résultats aux travaux de Baumol (1968). Ainsi nous pouvons positionner les décideurs non routiers entre les créateurs gestionnaires et les créateurs entrepreneurs. Nous qualifions ces derniers d'entrepreneurs proactifs. De tels entrepreneurs proactifs déclarent avoir totalement confiance en eux et se considèrent comme fortement innovants. Ils associent les modalités relatives à l'autonomie et à la cognition. Leurs réponses aux questions liées à l'accomplissement sont concordantes à celles relatives au comportement dans l'action. Ils cherchent à aller juste qu'au bout des projets qu'ils initient peu importent les difficultés et saisissent souvent les occasions qui se présentent. Ils tentent à mettre tout en oeuvre pour augmenter leur chance de réussite et refusent l'échec. Inversement, les entrepreneurs routiniers évaluent faiblement les questions liées à la confiance en soi et celles relatives à l'innovation. Ils s'efforcent très peu de connaître les nouveautés de leur domaine d'activité. Les projets exigeants les découragent au vu des réponses fournies aux questions liées à la persévérance et à l'accomplissement. Ils limitent le nombre d'activités à accomplir simultanément et avouent avoir du mal à appréhender les occasions d'affaires dans les événements qui surviennent dans leur environnement.

Il nous semble qu'à travers les interactions avec des éléments de son champ d'intérêt et d'aptitudes, l'entrepreneur apprend à observer, à agir, à réagir, à discerner, à anticiper des comportements et à adapter ses objectifs et ses actions. C'est dans l'action complexe 
orientée par la recherche d'efficacité satisfaisante, d'indépendance, de coopération, de domination, évitant des menaces perçues et profitant d'opportunités éphémères, que l'entrepreneur réactualise à dessein ou instinctivement sa "connaissance" du monde qui l'entoure. Ses représentations se font et se défont au fur et à mesure que les résultats de ses interactions avec les entités distinctes de son champ d'intérêt. Autrement dit, ses prédispositions cognitives et psychologiques confrontées à ses expériences quotidiennes confirment, infirment ou altèrent les représentations qu'il se fait de ses caractéristiques entrepreneuriales, qui guident ses actions et leur donnent une persistance, une réorientation ou une érosion. Le démarrage, la survie, la performance et la croissance de son entreprise deviennent alors tributaires de la vitesse et de la manière dont le couplage Entreprise-Environnement sera assuré du fait des caractéristiques entrepreneuriales.

\section{Perspectives de recherche: l'incontournable pédagogie d'accompagnement}

La perception des caractéristiques entrepreneuriales met en évidence l'importance d'une démarche incitative voire démonstratrice d'un système de compétences structuré et explicatif de type de décision: routinier ou non routinier. Le cheminement que peut effectuer le créateur gestionnaire (ou exclusivement routinier) vers le créateur entrepreneur (ou véritablement non routinier) doit encourager l'apprentissage dans une perspective systémique. La technique ou la pédagogie d'accompagnement souhaitée est révélatrice d'un état d'esprit de progrès et de mise en relation avec les caractéristiques entrepreneuriales de base. Il apparaît qu'avec le dispositif d'accompagnement, les valeurs et attitudes désirées se nourrissent des méthodes de structuration des compétences entrepreneuriales, d'analyse stratégique et de gestion de l'innovation. Ces méthodes apparaissent comme des compléments indispensables à l'intuition managériale des futurs créateurs. Ils sont conscients du chemin à parcourir en termes de connaissance soustendue par des caractéristiques particulières. Celles-ci favorisent une certaine emprise sur l'environnement souvent multiforme et changeant.

Dans cette optique, l'accompagnateur, à partir de ses missions (de conseil, de formateur et de concepteur d'outils ou de modèles) doit jouer un double rôle (Baron, 1989, cité par Stern et Tutoy, 1998): le rôle de réserve d'énergie (apport au niveau de la méthode, du choix de projet stratégique, de l'explication et de la mise en œuvre de ce projet) et celui de catalyseur (développement d'une opération de changement, notamment culturel). Alors, il peut introduire progressivement des changements au niveau de la vision stratégique du dirigeant créateur, de ses méthodes, de ses conduites et du système organisationnel dans son ensemble. Il est également clair que le point d'entrée de chaque programme d'accompagnement doit rester spécifique. L'ouverture à d'autres compétences comporte des limites qui doivent être étudiées et cernées par les acteurs du réseau mis en place. On sait que l'introduction d'une innovation qui conduit à de nouveaux produits, process ou à de nouveaux marchés fait appel à la plupart des études et des conditions non courantes. L'approche se veut souvent globale ou systémique avec les dimensions cognitives, psychologiques et sociologiques de l'environnement de l'entrepreneur qui se positionne dans un statut contextuel et intuitif.

D'un côté, du fait des phénomènes perceptifs, l'environnement de l'entrepreneur n'est pas figé à l'image instantanée du réel et contient des potentialités d'action et de réflexion qui ne coïncident pas forcément avec d'autres observations intervenant parallèlement dans le même espace /temps. L'environnement entrepreneurial paraît comme une construction virtuelle à l'image des potentialités que révèle l'activité cognitive des acteurs et que 
nourrissent les interactions quotidiennes de leurs actions. Lors de cette construction, le système de représentations qui se nourrit de perpétuels construits externes joue le rôle de filtre et de générateur d'idées en même temps.

D'un autre côté, du fait de la complexité qui entoure l'entrepreneur, des domaines cruciaux de l'environnement sont détectés en rapport avec un espace d'interactions, des entités distinctes qui l'occupent, la nature des interactions qui l'animent et le temps nécessaire pour percevoir, interpréter et incorporer les changements. L'entrepreneur les organise en fonction des représentations qu'il en fait et des attentes perçues. Il s'agit d'un environnement en construction/destruction circulaire, aussi vrai que les multiples interprétations que se font des décideurs agissant dans des conditions réelles, singulières incertaines et non linéaires.

\section{REFERENCES BIBLIOGRAPHIQUES}

[1] Abric J-C, Pratiques sociales et Représentations, Puf, 1er édition, Paris, 1994

[2] Acs, Z.J. and Audretsch, D.B., Handbook of Entrepreneurship Research. An Interdisciplinary Survey and Introduction. Dordrecht: Kluver Academic Publishers, 2003

[3] Amit, R., McRimmon, K.R., Zietsma, C. and Oesch, J.M., "Does money matter? Wealth attainment as the motive for initiating growth-oriented technology ventures", Journal of Business Venturing, 16, 2000, p. 119-143.

[4] .Audretsch, D. Entrepreneurship: A Survey of the Literature. Paper Prepared for the European Commission, 2002, Enterprise Directorate General.

[5] Barreto, H., The Entrepreneur in Micro-economic Theory: Disappearance and Explanation. New York: Routledge, 1989.

[6] Baumol, W.J., "Entrepreneurship in economic theory", American Economic Review, 58(2), 1968, p. 64-71.

[7] Blanchflower, D. and Oswald, A., "What makes a young entrepreneur?" LSE Centre of Labour Economics, Discussion Paper 373. 1990

[8] Bull, I., Thomas, H. and Willard, G., Entrepreneurship: Perspectives on Theory Building. London: Elsevier, 1996.

[9] Casson, M.. The Entrepreneur. An Economic Theory. Cheltenam: Edward Elgar Publishing, 1982

[10] Casson, M., The Entrepreneur. An Economic Theory. Second Edition. Northampton: Edward Elgar Publishing, 2003.

[11] Crozier M., L'entreprise à l'écoute, Inter-Edition, Paris, 1989.

[12] Day, R.H. and Sunder S., "Review: Ideas and work of Richard M. Cyert". Journal of Economic Behaviour and Organization, 31, 1996, p. 139-148

[13] De Fraja, G., "Entrepreneur or manager: Who runs the firm?" Journal of Industrial Economics, 44(1), 1996 p. 89-98

[14] Dearborn D. C. et Simon H. A., "Selective Perception: A Note on the Departmental identification of Executives" Sociometry, Vol. 21, 1958, pp. 140-144

[15] Durkheim E., «Représentations individuelles et représentations collectives » Revue métaphysique et morale, VI 1898, p. 273-302

[16] Eboli, M, Firms' performance under uncertainty: the entrepreneur as a forecaster. ISFSE, 1997

[17] Eisenhardt. M., «Le manager lent et le manager rapide. Comment accélérer les processus décisionnels » Gérer et comprendre, Septembre 1992, p. 4-18.

[18] Gasse Y., Evaluation des caractéristiques entrepreneuriales, Québec, Québec, Les éditions de la fondation de l'entrepreneurship, 1996.

[19] Gasse Y., «L'influence du milieu dans la création d'entreprises », Revue Organisations et Territoires, vol. $12, \mathrm{n}^{\circ} 2,2003$, p.49-54.

[20] Gartner, W.B., "What are we talking about when we talk about entrepreneurship?" Journal of 
Business Venturing, 5(1), 1990, p. 15-28.

[21] Glancey, K.S. and McQuaid, R.W., Entrepreneurial Economics. London: Macmillan Press, 2000

[22] Goffee, R. and Scase, R. Entrepreneurship in Europe: The Social Process. London: Croom Helm, 1987

[23] Iyigun, M.F. and Owen, A.L., "Risk, entrepreneurship, and human-capital accumulation" American Economic Review, 88(2), 1998, p. 454-457.

[24] Kihlstrom, R.E. and Laffont, J.J., "A general equilibrium entrepreneurial theory of firm formation based on risk aversion". Journal of Political Economy, 87(4), 1979 p. 719-748.

[25] Kirzner, I.M., Entrepreneurship and the future of capitalism. In Bachman, J. (ed.). Entrepreneurship and the Outlook for America. New York: The Free Press, 1983.

[26] Knight, F.H., Risk, Uncertainty and Profit. New York: Harper and Row, 1921.

[27] Leibenstein, H., "Allocative efficiency vs. 'X-efficiency", American Economic Review, 56, 1966, P.392-415.

[28] Leibenstein H., "A branch of economics is missing: Micro-macro theory". Journal of Economic Literature, 17(2), 1979, p. 477-502.

[29] LE Moigne J. L., Les épistémologies constructivistes, PUF, Paris, 1995.

[30] Mintzberg H., Le pouvoir dans les organisations, Ed. Organisation, Paris, 1986

[31] Moles A., Théories des actes. Vers une écologie des actions, Casterman, Paris, 1977

[32] Moles A., Les sciences de l'imprécis, Ed. du Seuil, Paris, 1990.

[33] Moscovici S., La psychanalyse : son image et son public, PUF, Paris, 1961

[34] Peters T. et Waterman R., Le prix de l'excellence, InterEditions, Paris, 1983

[35] Pfeffer J. et Salancik G. R., The External Control of Organisations: A ressource dependence perspective, Harper \& Row, New York, 1978

[36] Raux J. F., «L'entreprise entre incertitude et connaissance » Revue Internationale de Systémique, Vol. 9, n², 1995, p. 199-213.

[37] Ricketts, M., The Economics of Business Enterprise: An Introduction to Economic

[38] Organization and the Theory of the Firm. Ashgate: Edward Ellgar, 2002

[39] Schumpeter, J. A., The Theory of Economic Development. Second edition. Cambridge: Harvard University Press, 1936

[40] Schumpeter, J.A., Capitalism, Socialism and Democracy. London: Uniwin, 1942

[41] Shane, S.A., Prior knowledge and the discovery of entrepreneurial opportunities. Organization Science, 4, 2000, p. 448-469.

[42] Simon H. A., «Strategy and Organizational Evolution » Strategic Management Journal, Special isuue, Winter 1993, p. 131-142.

[43] Stacey R.D., "The Science of Complexity : An Alternative Perspective for Strategic Change Processes » Strategic Management Journal, Vol.16, 1995, p. 477-195.

[44] Stern P. et Tutoy P., Le métier de consultant : principes, méthodes et outils, Les Editions D’Organisation, Paris 1998

[45] Storey, D., Small Business: Critical Perspectives on Business and Management. London: Routledge, 2000

[46] Swedberg, R., Entrepreneurship: The Social Science View. New York: Oxford University Press, 2000

[47] Westhead, P. and Wrigth, M., Advances in Entrepreneurship. Cheltenam:

[48] Edward Elgar, 2000

[49] Zaoual H., Du rôle des croyances dans le développement économique » Thèse de doctorat, Université des Sciences et Technologies de Lille, Lille 1, 1996 


\section{ENTREPRENEURSHIP AND DECISIVENESS OF A MANAGER- A CREATOR OF AN ENTERPRISE}

Concerning decision making within organizations, there is a particular person who creates his own business and ensures its' functioning and sustainability. It is therefore necessary to investigate personal creative entrepreneurial characteristics as determinants of decision making. We can do this by working on the inventory of entrepreneurial characteristics (Gasse, 1996; 2003). Entrepreneurial characteristics allow us to identify the entrepreneurial potential of each creator. Further research leads to their use as determinants of creative decision making, which is the subject of this article. The characteristics of entrepreneurs were identified as to how they made the choice of starting a business from their own perceptions or self-image. This approach has been adapted to a survey of 172 executives of SMEs in creative activity regarding business sustainability in the last quarter of 2009. The essential purpose of such a survey is to make an inventory and classify entrepreneurial characteristics and, subsequently, put forward a typology of entrepreneurs. Given the characteristics of the survey, the analysis differentiates the perception of their decision to becoming an entrepreneur.

The results of our study lead us to three categories of entrepreneurs given their opinions concerning the characteristics that determine their behavioral decision making. The results were compared to the work of Baumol (1968). In this way we can differenciate creative entrepreneurs and creative managers hence the study path for creator managers or creative entrepreneurs can be differenciated enabling development of appropriate teaching methods and enabling teachers to provide appropriate guidance.

Keywords: Entrepreneurial characteristics, motivation, decision, values, entrepreneurship

DOI: 10.7862/rz.2015.mmr.5

Tekst złożono w redakcji: kwiecień 2015

Przyjęto do druku: kwiecień 2015 